\title{
Distinguishing benign confounding treatment changes from residual prostate cancer on MRI following laser ablation
}

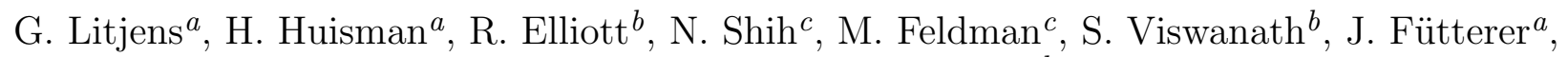 \\ J. Bomers ${ }^{a}$ and A. Madabhushi ${ }^{b}$ \\ ${ }^{a}$ Radboud University Nijmegen Medical Centre, Nijmegen, The Netherlands; \\ ${ }^{b}$ Case Western Reserve University, Cleveland, USA; \\ ${ }^{c}$ University of Pennsylvania, Philadelphia, USA;
}

\begin{abstract}
Laser interstitial thermotherapy (LITT) is a relatively new focal therapy technique for the ablation of localized prostate cancer. However, very little is known about the specific effects of LITT within the ablation zone and the surrounding normal tissue regions. For instance, it is important to be able to assess the extent of residual cancer within the prostate following LITT, which may be masked by thermally induced benign necrotic changes. Fortunately LITT is MRI compatible and hence this allows for quantitatively assessing LITT induced changes via multi-parametric MRI. Of course definite validation of any LITT induced changes on MRI requires confirmation via histopathology. The aim of this study was to quantitatively assess and distinguish the imaging characteristics of prostate cancer and benign confounding treatment changes following LITT on 3 Tesla multi-parametric MRI by carefully mapping the treatment related changes from the ex vivo surgically resected histopathologic specimens onto the pre-operative in vivo imaging. A better understanding of the imaging characteristics of residual disease and successfully ablated tissue might lead to improved treatment monitoring and as such patient prognosis. A unique clinical trial at the Radboud University Medical Center, in which 3 patients underwent a prostatectomy after LITT treatment, yielded ex-vivo histopathologic specimens along with pre- and post-LITT MRI. Using this data we (1) identified the computer extracted MRI signatures associated with treatment effects including benign necrotic changes and residual disease and (2) subsequently evaluated the computer extracted MRI features previously identified in distinguishing LITT induced changes in the ablated area relative to the residual disease. Towards this end first a pathologist annotated the ablated area and the residual disease on the ex-vivo histology and then we transferred the annotations to the post-LITT MRI using semi-automatic elastic registration. The pre- and post-LITT MRI were subsequently registered and computer-derived multi-parametric MRI features extracted to determine differences in feature values between residual disease and successfully ablated tissue to assess treatment response. A scoring metric allowed us to identify those specific computer-extracted MRI features that maximally and differentially expressed between the ablated regions and the residual cancer, on a voxel-byvoxel basis. Finally, we used a Fuzzy C-Means algorithm to assess the discriminatory power of these selected features. Our results show that specific computer-extracted features from multi-parametric MRI differentially express within the ablated and residual cancer regions, as evidenced by our ability to, on a voxel-by-voxel basis, classify tissue as residual disease. Additionally, we show that change of feature values between pre- and postLITT MRI may be useful as a quantitative marker for treatment response (T2-weighted texture and DCE MRI features showed largest differences between residual disease and successfully ablated tissue). Finally, a clustering approach to separate treatment effects and residual disease incorporating both (1) and (2) yielded a maximum area under the ROC curve of 0.97 on a voxel basis across 3 studies.
\end{abstract}

Keywords: Laser ablation therapy, prostate cancer, MRI, treatment response

\section{INTRODUCTION}

Radical treatment of prostate cancer is known for its relatively high incidence of side-effects like incontinence and impotence. ${ }^{1}$ Furthermore, most prostate cancer is not aggressive and might not warrant radical therapy. This has spurred substantial interest in less radical, localized therapy options. Examples are cryo-ablation, high-intensity focused ultrasound or laser-ablation therapy. ${ }^{2,3}$ Laser interstitial thermotherapy (LITT) is one such focal therapeutic procedures which is compatible with magnetic resonance imaging (MRI), allowing for

Medical Imaging 2014: Image-Guided Procedures, Robotic Interventions, and Modeling,

edited by Ziv R. Yaniv, David R. Holmes III, Proc. of SPIE Vol. 9036, 90361D

(C) 2014 SPIE · CCC code: $1605-7422 / 14 / \$ 18 \cdot$ doi: $10.1117 / 12.2043819$

Proc. of SPIE Vol. 9036 90361D-1 
high resolution in vivo imaging to be used in LITT procedures for treatment guidance to the index lesion. ${ }^{4}$ Furthermore, from previous studies on LITT for liver lesions, we know that the extent of tissue necrosis postLITT is visible on MRI. ${ }^{5}$ However, there is very little work describing the imaging characteristics of LITT-induced changes in the prostate. ${ }^{6}$ Understanding changes in MRI features post-LITT is important for accurate follow-up of the patient because it will allow the clinician to assess whether the ablation was successful (i.e. whether all the primary tumor has disappeared). If residual disease is present, the area could be re-ablated or a different therapy option could be chosen. Furthermore, qualitative observations of LITT-related changes on prostate MRI do not specifically address how to differentiate between the appearance of benign LITT-related changes (e.g. edema, necrosis) that can mask the presence of residual cancer, post-LITT. This implies a need for co-registration and image analysis methods to quantitatively compare pre- and post-LITT MRI in order to identify voxel-by-voxel changes in MRI parameters that can describe LITT-related changes within the prostate.

Treatment evaluation of therapeutic options for prostate cancer have primarily been examined for radiation treatment in a number of qualitative studies. ${ }^{7,8}$ For LITT, a Phase I trial found good correlation between volumes of thermal damage that were visible on MRI and those determined via staining of ex vivo surgical prostatectomy specimens from patients who had previously undergone LITT. ${ }^{9}$ Additionally while the ablated volume measured on MRI was marginally overestimated compared to pathology, MR images demonstrated excellent capability in discriminating non-viable necrotic tissue, post-ablation.

However, for imaging changes between pre- and post-LITT MRI, only Viswanath et al. investigated the imaging characteristics of LITT related changes on $\mathrm{MRI}^{6}$ following treatment for prostate cancer. While they found changes in imaging markers following LITT and specifically found that there were particular imaging markers that revealed more dramatic changes compared to other markers, all the analysis was limited to the ablation zone. Since no histopathology was available, it was not possible to rigorously evaluate whether the changes in imaging markers were driven by treatment effects or by residual disease. Additionally, the lack of histopathology meant that it was not possible to evaluate whether the residual disease had itself undergone any imaging changes and whether these changes were discernible on MRI.

The aim of this study was to quantitatively assess and distinguish the imaging characteristics of prostate cancer and benign confounding treatment changes following LITT on 3 Tesla multi-parametric MRI by uniquely taking advantage of available ex vivo surgically resected histopathology specimens. A unique clinical trial at the Radboud University Medical Center, in which 4 patients underwent a prostatectomy after LITT treatment, yielded ex-vivo histopathologic specimens along with pre- and post-LITT MRI. Using this data we (1) identified the computer extracted MRI signatures associated with treatment effects and residual disease following LITT on MRI, (2) subsequently evaluated the previously identified imaging signatures in distinguishing LITT induced changes changes in the ablated area relative to the residual disease on MRI. A flowchart detailing the pipeline and methodology is shown in Figure 1.

To investigate (1), we first need to identify treatment effects and residual disease on histology and subsequently establish a voxel correspondence between the post-LITT MRI and the histology. To this end first the histology was annotated by a pathologist, after which we performed a careful semi-automatic elastic registration of the histology to the post-LITT MRI. ${ }^{10}$ Finally, the histology annotations were propagated to the post-LITT MRI and computer-extracted features were obtained (Table 1). In this work computerized feature analysis in multi-parametric prostate MRI was designed in a way that allows for optimally expressing each individual MR parameter, as each parameter characterizes different behavior within the underlying tissue. Multi-parametric prostate MRI typically consists of T2-weighted, diffusion-weighted and dynamic contrast-enhanced imaging. ${ }^{11,12}$ The T2-weighted imaging is mostly used for its high resolution and contrast, allowing detailed visualization of the tissue anatomy. From clinical guidelines we know that the T2-weighted images are especially useful to assess the texture of prostate lesions. ${ }^{12}$ Prostate cancer exhibits a so-called 'erased charcoal sign', a smudge-like dark texture on $\mathrm{T} 2$-weighted images ${ }^{12}$ and previous work has suggested that ablated areas have different textured appearance compared to normal or benign tissue. ${ }^{6}$

Diffusion weighted imaging is specifically useful for characterizing tissue at a microscopic level, enabling us to assess traits like cell density at the MRI level. In diffusion weighted imaging, several images with different b-values are acquired. ${ }^{11,13}$ An increased b-value implies increased diffusion-weighting. Furthermore, to remove protocol dependency an apparent diffusion coefficient map is calculated, which is a roughly quantitative measure 
of tissue diffusivity. Prostate cancer has a high cellular density compared to the normal glandular structure of the prostate. This results in a reduced diffusivity in cancerous tissue and thus a high signal in high b-value images and subsequently a lower apparent diffusion coefficient values. LITT tends to cause necrosis and formation of scar tissue and might thus alter diffusivity in the tissue. Furthermore, prostate cancer lesions tend to have a focal appearance on diffusion-weighted imaging, the shape of which might change due to therapy.

Finally, dynamic contrast enhanced MRI allows for visualization of the uptake of contrast agent in tissue. This allows us to measure attributes of the tissue vasculature, like the relative fraction of extra-vascular, extracellular space in each voxel and micro-vessel permeability. ${ }^{14}$ Prostate cancer lesions tend to have leaky microvasculature, which results in fast initial enhancement and wash-out. Additionally, inflammation and tissue death (necrosis/apoptosis) caused by the treatment might cause blood flow and vascular changes in the ablated area.

To extract feature changes to assess their potential in discriminating successfully ablated tissue and residual disease (2), we first need to register the pre- and post-LITT MRI to establish voxel correspondence. We then extract computer derived image features for characterizing treatment related changes within the ablation zone on pre-LITT MRI. Subsequently, a feature score is calculated to identify features which changed most in treatment area, relative to the residual disease. This allows us to, on a voxel-by-voxel level discriminate between feature changes in residual disease and successfully ablated tissue. After identifying the features that reveal the largest differential change between the residual cancer and benign thermally changed regions, a fuzzy C-means clustering algorithm is used to evaluate the performance of the computer extracted features on MRI.

The structure of this paper is as follow: Section 2 will describe the data and the experimental design. In Section 3 we will assess and discuss the results of our experiments. Last, in Section 4 we will come back to the initial hypothesis and offer some concluding remarks.

\section{EXPERIMENTAL DESIGN}

(a)

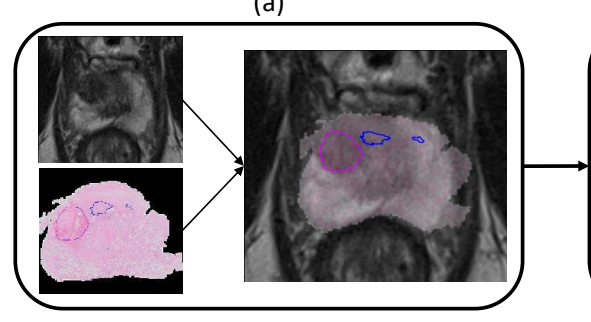

(b)
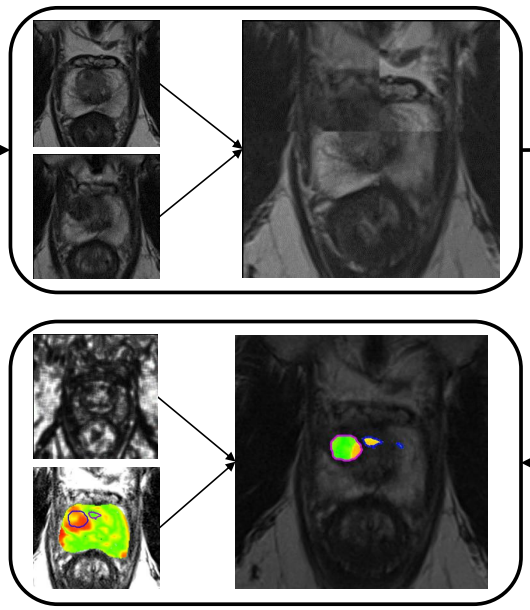

(e) (c)

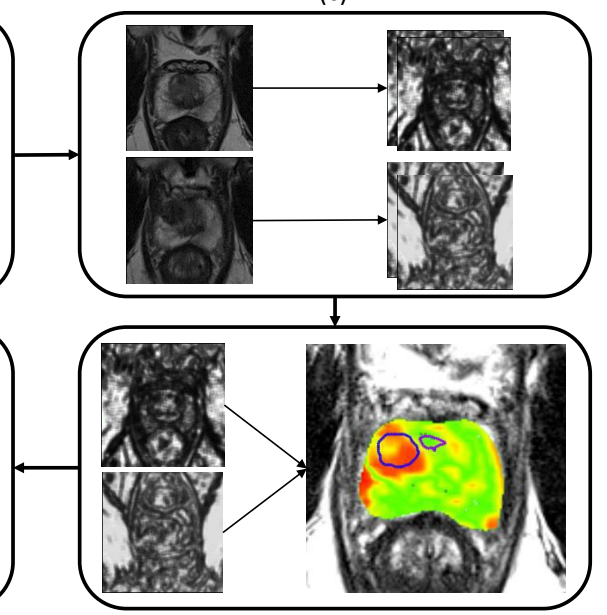

(d)

Figure 1. Flowchart detailing the process of (a) co-registration of histopathology and MRI using a semi-automated thin plate spline approach, (b) registration of pre- and post-LITT MRI using affine registration with localized mutual information, (c) extraction of features (Table 1) and (d) calculation of relative feature change and finally (e) the clustering result to detect residual disease using fuzzy C-means clustering.

\subsection{Data description}

Four patients underwent both pre- and post-LITT multi-parametric MRI (3 Tesla, Siemens Skyra MR scanner). All studies were acquired with only a pelvic-phased array coil, except the post-LITT MRI study of one patient, 
which was acquired with an endorectal coil. After the post-LITT MRI all patients underwent a radical prostatectomy. Subsequently, the slide with the largest ablated area was digitized using an Olympus digital slide scanner at 20X. An experienced pathologist annotated areas of LITT induced changes and residual disease on the prostatectomy slide including the largest ablated area. One patient was subsequently excluded because no residual disease was present. Both the pre- and post-LITT MRIs consisted of T2-weighted imaging, a diffusion-weighted sequence including three b-values $(50,400,800)$, a dynamic-contrast enhanced time series (36 time points, 4 seconds temporal resolution) and a proton density-weighted image. The scanner software calculated an apparent diffusion coefficient (ADC) based on the diffusion-weighted imaging.

\subsection{Histology/MRI registration}

The pathology annotations were transferred to the post-LITT MRI by establishing slice correspondences and subsequently registering the whole-mount slide to the post-LITT MRI by using a thin plate spline registration technique. ${ }^{15}$ The process, in a step-by-step fashion, goes as follows:

1. A pathologist annotated the areas of residual disease and successfully ablated tissue on the whole mount prostatectomy slide using a contouring tool.

2. The slice in the MRI which corresponds to the prostatectomy slide was established by an image analysis researcher under the supervision of a radiologist by comparing landmarks on the prostate boundary on the pathology and the MRI.

3. Corresponding points were indicated on the prostate boundary for both the prostatectomy slide and the MRI slice.

4. A thin plate spline transformation was calculated to move from the prostatectomy coordinate space to MRI coordinate space. ${ }^{16}$

5. The histopathology image was transformed to the MRI space using this b-spline transformation and a visual assessment of registration quality was made.

6. The annotations of the pathologist were morphed to the MRI using this b-spline transformation.

7. The pre-LITT MRI was subsequently registered (affine/elastic) to the post-LITT MRI to establish voxel correspondence (Figure 1(a))

An example of the results from this process are show in Figure 2.

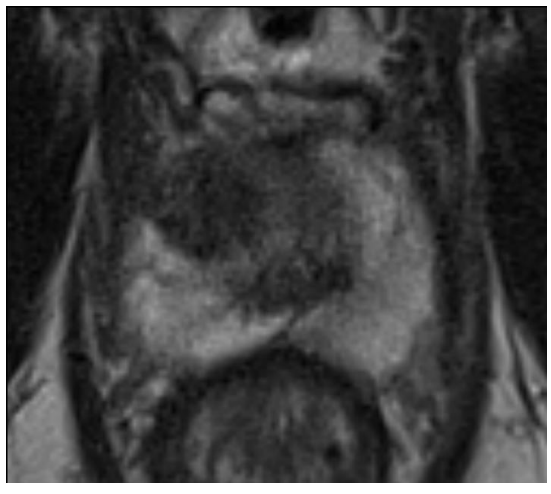

(a)

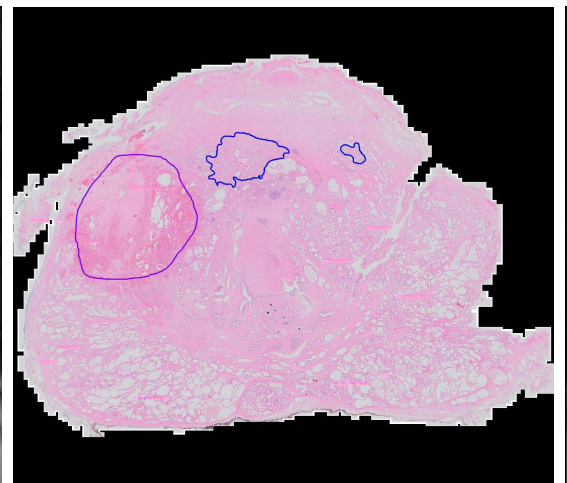

(b)

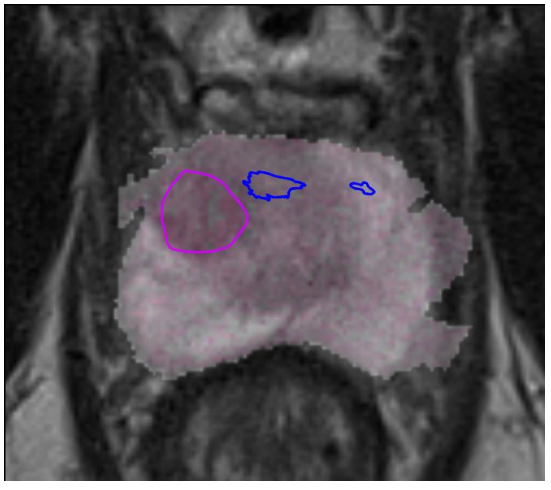

(c)

Figure 2. Example images of the post-LITT MRI (a) and a H\&E stained prostatectomy slide (b) and the result of the subsequent MRI/histology registration (c). Ablated area in purple, residual disease in blue 


\begin{tabular}{|c|c|c|c|}
\hline Category & Feature name & MR parameter & Feature settings \\
\hline Intensity & $\begin{array}{l}\text { (Pseudo)T2-map }^{18} \\
\text { ADC } \\
\text { b- } 800\end{array}$ & $\begin{array}{l}\text { T2W } \\
\text { DWI } \\
\text { DWI }\end{array}$ & \\
\hline Texture & $\begin{array}{l}\text { 2D Multi-scale Gaussian Derivatives } \\
\text { 2D Haralick texture measures } \\
\text { 2D } \\
\text { 2D Multi-angle Gabor } \\
\text { 2D Li Multi-scale blobness }\end{array}$ & $\begin{array}{l}\text { T2Map } \\
\text { T2Map } \\
\text { T2Map } \\
\text { T2Map, ADC, b800, } \\
\text { Ktrans, Kep, Ve, time-to-peak, } \\
\text { maximum enhancement, } \\
\text { wash-out rate }\end{array}$ & $\begin{array}{l}\text { Up to } 2 \text { nd order, } \sigma=2.0,2.67,4.1 \text { and } 6.0 \mathrm{~mm} \\
\text { Kernel sizes } 3,5,7 \text { voxels } \\
\text { Four angles: } 0, \frac{\pi}{4}, \frac{\pi}{2}, \frac{3 \pi}{4}, \lambda=1.5,2 \text { and } 4 \text { voxels } \\
\sigma=2.0,2.67,4.1 \text { and } 6\end{array}$ \\
\hline Pharmacokinetic & $\begin{array}{l}\text { Curve fitting parameters }{ }^{20} \\
\text { Std. Tofts PK model }{ }^{20}\end{array}$ & $\begin{array}{l}\mathrm{DCE} \\
\mathrm{DCE}\end{array}$ & $\begin{array}{l}\text { Time-to-peak, maximum enhancement, wash-out rate } \\
\text { Ktrans, Kep, Ve }\end{array}$ \\
\hline
\end{tabular}

Table 1. Summary of features and feature settings calculated for both the pre- and post-LITT MRI

\subsection{Pre-LITT/Post-LITT MRI registration}

To establish voxel correspondences between the post- and pre-LITT MRI we used the Elastix registration software. ${ }^{17}$ For two out of three patients we applied an affine registration, for one patient we applied an elastic registration because the post-LITT MRI was acquired with an endorectal coil, whereas the pre-LITT MRI was acquired with only a pelvic phased array coil. Registration was performed in two steps, first a translation component was estimated after which either the affine transformation matrix was determined or the b-spline elastic transformation grid. Localized mutual information was used as a similarity metric to drive the registrations. An example results is shown in Figure 3.

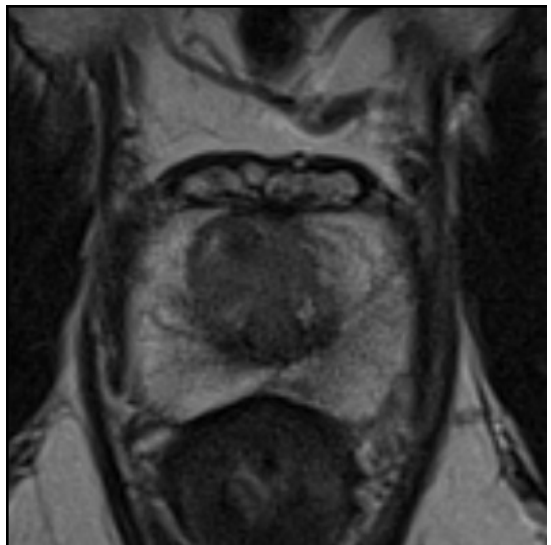

(a)

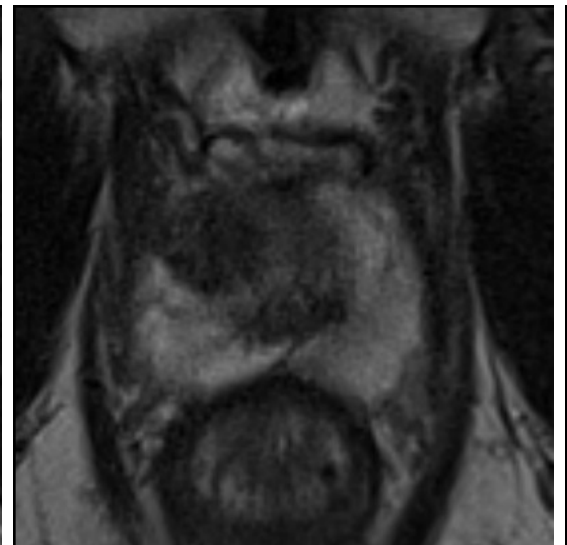

(b)

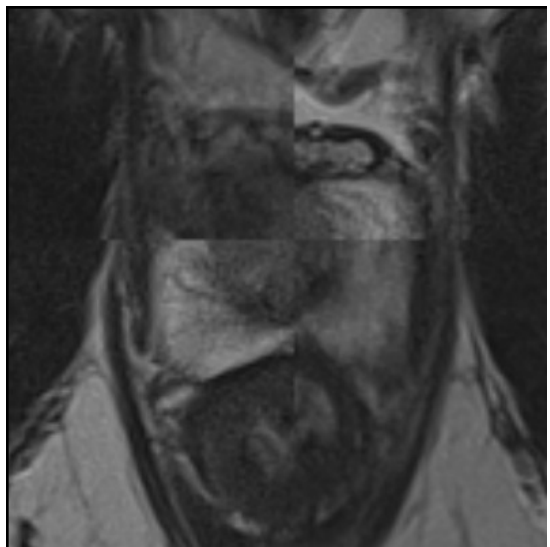

(c)

Figure 3. Example images of the pre-LITT MRI (a), post-LITT MRI (b), and the subsequent affine registration result in a checkerboard form. Registration was performed using localized mutual information as a metric.

\subsection{Feature calculation and scoring}

In total 93 features were extracted to establish the imaging characteristics of benign confounding treatment changes and residual disease. An overview of all features used in this study is given in Table 1.

\subsubsection{Intensity standardization}

Intensity drift is an issue that is well known in MRI. ${ }^{15}$ This means that intensities differ from scanner to scanner and even from protocol to protocol or scan to scan on the same scanner. Intensity drift might also have happened between the pre- and post-LITT images. To circumvent this issue in T2-weighted images we can calculate a (pseudo)T2-map using the transverse T2W-image and the proton density-weighted image as described in. ${ }^{21}$ This approach uses MR signal equations and a muscle reference region of interest to reduce intensity drift between the pre- and post-LITT acquisition. 


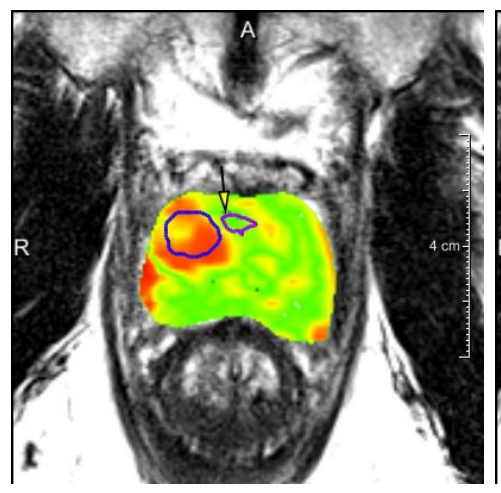

(a)

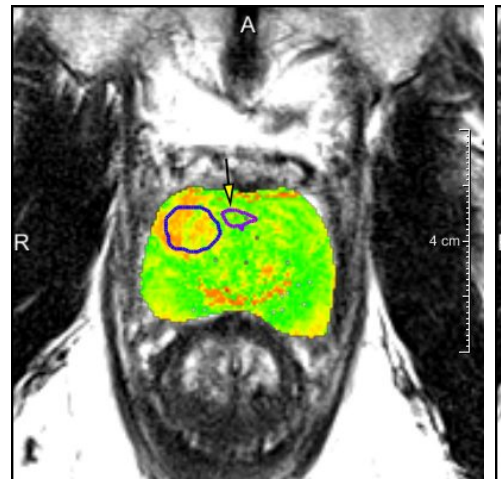

(c)

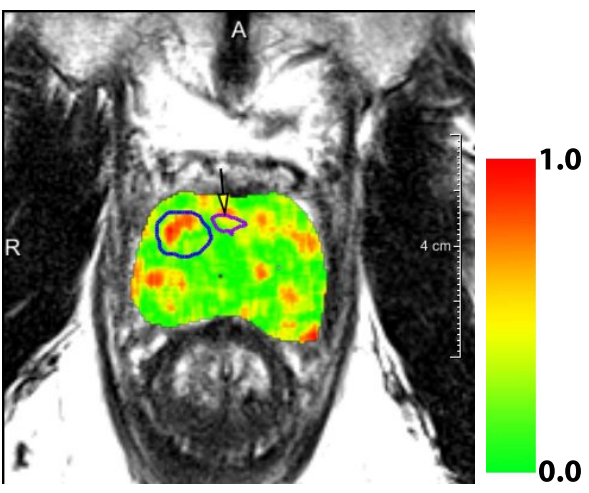

(b)

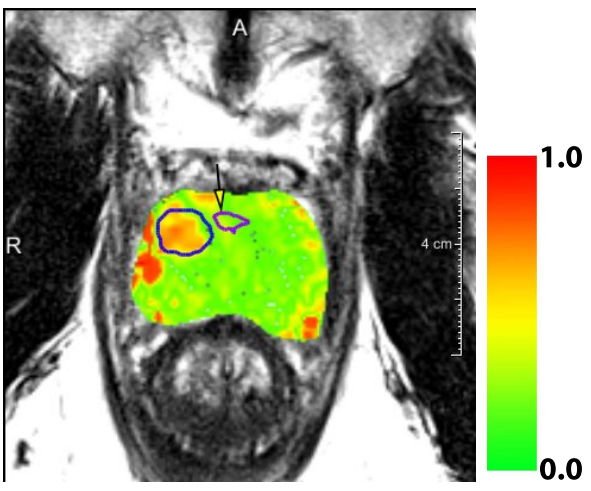

(d)

Figure 4. Overlays of normalized feature change between pre- and post-treatment MRI. Figures a, b, c and d represent the features $K^{\mathrm{ep}}$, Haralick Correlation, (Pseudo)T2-Map and $V_{e}$ respectively. In the overlay red indicates areas of high relative change and green of low relative change. The successfully ablated area is indicated in a blue contour and the purple contour indicates residual disease. The residual disease area is also indicated with a yellow arrow.

\subsubsection{T2-weighted imaging features}

For the T2-weighted image, we calculated several texture features. From previous studies we know that there exists a difference in texture between normal prostate tissue and LITT therapy effects. ${ }^{6}$ Furthermore, there are distinct texture differences between prostate cancer and normal prostate tissue. ${ }^{12}$ We hypothesize that their thus also must exist texture difference between residual prostate cancer and bening LITT related changes. We used 13 Haralick texture features using 3 kernel sizes (3,5 and 7 voxels), Gabor texture features using 4 different angles and 3 different wavelengths between $1 \mathrm{~mm}$ and $6 \mathrm{~mm}$ and Gaussian derivatives up to second order using 4 different scales between $1 \mathrm{~mm}$ and $6 \mathrm{~mm} .{ }^{15}$ The texture features were all calculated on the (pseudo)T2-map.

\subsubsection{Diffusion-weighted imaging features}

For the diffusion-weighted imaging we directly used the ADC values and the b800 image intensities. To take advantage of the fact that prostate lesions tend to exhibit a focal appearance on diffusion-weighted imaging and that treatment effects may not show this feature we implemented the multi-scale blobness filter proposed by Li et al. ${ }^{22}$ and calculated the filter using 4 scales between 1 and $6 \mathrm{~mm}$ on the b800 and ADC images.

\subsubsection{Dynamic contrast-enhanced imaging features}

Dynamic contrast-enhanced MRI also tends to suffer from scanner and protocol dependency. To remove this dependency and extract the most useful information from these curves we implemented curve fitting and pharmacokinetic modeling routines as presented in. ${ }^{20,23,24}$ The curve fitting routine uses a bi-exponential curve model and was implemented to enable faster and more robust pharmacokinetic modeling. The pharmacokinetic model 


\begin{tabular}{l|l|c|c|c}
\hline & & \multicolumn{3}{|c}{ Median normalized relative change in } \\
Rank & Feature & Residual disease & Treated area & MRI parameter \\
\hline 1 & Kep & 0.05 & -0.11 & DCE \\
2 & Gabor $\theta=0, \lambda=1.5$ & 0.07 & 0.14 & T2W \\
3 & Gauss. Deriv. $-\sigma=2.0$ & 0.08 & 0.17 & T2W \\
4 & T2Map & 0.07 & 0.12 & T2W \\
5 & Ktrans & 0.09 & 0.15 & DCE \\
6 & Gauss. Deriv. $-\sigma=2.8$ & 0.26 & 0.14 & T2W \\
7 & Haralick Correlation $($ ws $=7)$ & 0.09 & -0.40 & T2W \\
8 & Gabor $\theta=0.39, \lambda=1.5$ & 0.37 & 0.14 & T2W \\
9 & Gauss. Deriv. X $\sigma=6.0$ & -0.05 & 0.54 & T2W \\
10 & Time-to-peak & 0.07 & DCE \\
\hline
\end{tabular}

Table 2. The 10 top scoring features. Columns 3 and 4 show the normalized relative changes (Eq. 1.) in feature value between the pre- and post-LITT MRIs for both residual disease and treated area. It can be seen that all selected features are related to either T2W or DCE MRI. Furthermore, texture features show larger changes in both residual disease and successfully ablated tissue, however, the relative change is similar to the pharmacokinetic features.

that was implemented was the standard Tofts model, which neglects the vascular component in each voxel. The temporal resolution of the DCE time series was 4 seconds. To capture characteristics on the micro-vasculature we included total of 3 curve features (time-to-peak, washout rate and maximum enhancement) ${ }^{24}$ and 3 pharmacokinetic features $\left(K_{\text {trans }}, K^{\mathrm{ep}}, V_{e}\right) .{ }^{20}$ The micro-vasculature might be effected due to local tissue death and inflammation effects in the ablated area, as has been observed in for example the ablation of liver lesions. ${ }^{2}$ Furthermore, as cancer also tends to have a focal appearance on DCE MRI ${ }^{12}$ we also calculate the Li blobness filter on the $K_{\text {trans }}, K^{\mathrm{ep}}, V_{e}$, maximum enhancement, time-to-peak and wash-out rate images.

\subsubsection{Feature scoring}

Feature values may change both in the successfully ablated area and in the residual disease area. As such, feature which show a large change in one area and little in the other are most discriminative. We try to quantify this using a feature score. For each feature a score was established by calculating voxel differences between the pre- and post-LITT MRI in both the ablated and residual disease regions. A relative change per feature was calculated using:

$$
\begin{aligned}
d_{r}(f) & =\operatorname{median}_{c \in V}\left(\frac{f_{\text {post }}(c)-f_{\text {pre }}(c)}{f_{\text {pre }}(c)}\right) \\
S(f) & =\left.|| d_{r}(f)\right|_{\text {Res }}-\left|d_{r}(f)\right|_{\text {Abl }} \mid
\end{aligned}
$$

were $d_{r}$ is the relative change for feature $f$. V is the set of all voxels in a region and $c$ is a voxel. $f_{\text {post }}$ and $f_{\text {pre }}$ are feature $f$ on the post- and pre-LITT MRIs respectively. The score in equation 2 is then defined as the absolute difference between the relative change in the ablated area $d_{p}(f)_{\mathrm{Abl}}$ and in the residual disease $d_{p}(f)_{\text {Res }}$. This definition allows us to find features that are important in differentiating between residual disease and therapy effects. The median score over all patients was obtained as the overall score for that feature. Examples of features change is shown in Figure 4

\subsection{Clustering approach to discriminate residual disease from successfully ablated tissue}

A fuzzy C-means clustering to separate residual disease and treatment effects was performed for each patient. Clustering was chosen due to the limited amount of data (three patients) which we have in this study. A supervised classification approach typically tends to perform better than unsupervised methods like clustering, however only when enough training data is available to accurately train the classifier. Fuzzy C-means was chosen because it results in a likelihood of belonging to a cluster instead of a hard classification, which in turn allows us to evaluate the results using ROC analysis. The input to the clustering algorithm is unlabeled voxel feature data from the region encompassing both the ablated area and the residual disease. We repeat the clustering 


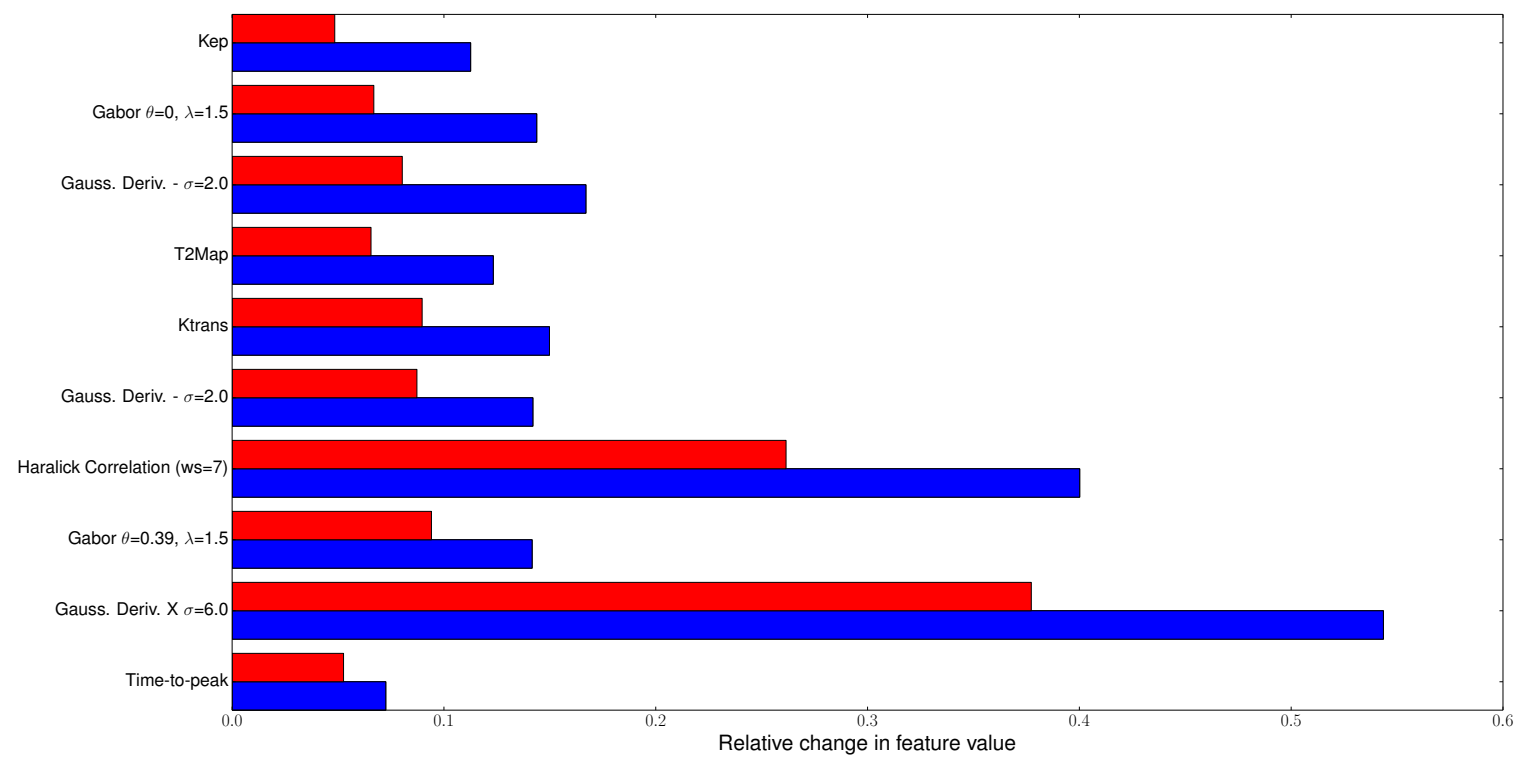

Figure 5. Relative change in feature value between residual disease (red) and the successfully ablated area (blue) for the top 10 scoring features (top to bottom). Almost all features are either pharmacokinetic $(1,5,10)$ or textural in nature $(2,3,6,7,8,9)$. Furthermore, although texture feature show more change in successfully ablated tissue relative to the pharmacokinetic features, they also show large change in the residual disease.

100 times with different initial cluster means to obtain the result with minimal root mean squared error. We perform the experiment with only the computer-extracted feature and subsequently repeat the experiment, with the addition of feature change of the top 10 scoring features as extra features.

\section{EXPERIMENTAL RESULTS AND DISCUSSION}

Our objectives were to identify features which (1) allow discrimination between residual disease and treatment effects and (2) showed the most treatment related change relative to residual disease. Finally, we wanted to incorporate this information to (3) improve detection of residual disease.

\subsection{Objective 1: What are there imaging characteristics specific for residual disease and treatment effects?}

For (1) we show in table 3, column 1 that our computer-extracted features are able to identify residual disease with an area under the ROC curve (AUC) up to 0.80. However, it can also be seen that this differs from patient to patient. This indicates that the differences between residual disease and successfully ablated area imaging characteristics cannot fully be explained by the appearance on the post-LITT MRI.

\subsection{Objective 2: Identify features that show discriminative change in successfully ablated tissue relative to residual disease.}

For objective (2), we present the 10 features with the highest feature change between pre- and post-LITT MRIs in Table 2 and Figure 5. One can appreciate that especially the dynamic contrast-enhanced MRI (positions 1, 5, and 10 in Table 2) and the T2-weighted texture features (positions 2, 3, 4, 6, 7, 8, and 9 in Table 2) show large differences between pre- and post-LITT MRI. We hypothesize that due to scar tissue formation in the ablated area the texture changes substantially because most of the scar tissue will be connective tissue, with few live cells. Compared to the residual disease, which will still have a lot of viable cancer cells, it is likely to result in distinct textures. Furthermore, blood flow and vascular content will be probably be markedly reduced in scar tissue. If we look at Table 2, we can see that the contrast agent transfer constant Kep is markedly reduced in the successfully ablated area, indicating reduced blood low. Furthermore, the time-to-peak has been 


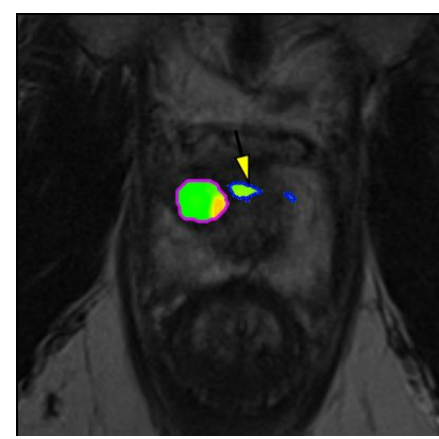

(a)

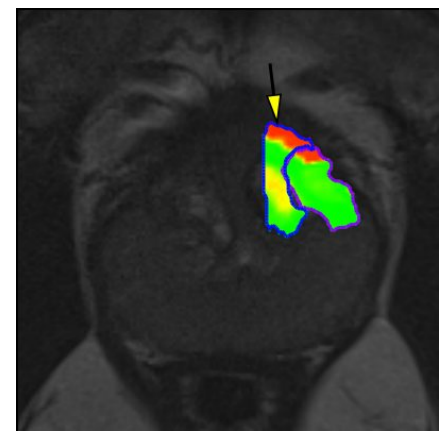

(c)

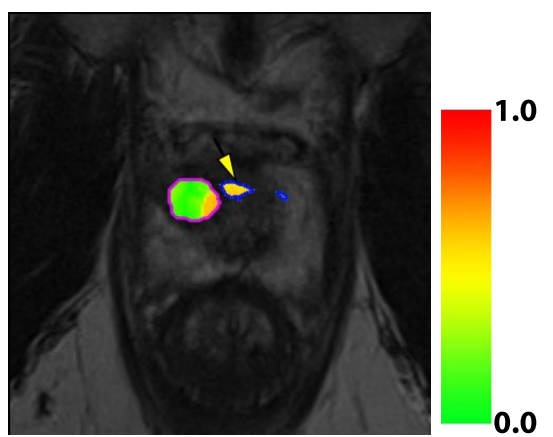

(b)

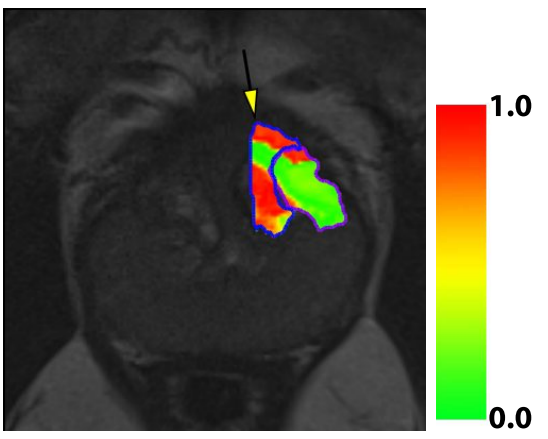

(d)

Figure 6. Likelihood heat maps for the fuzzy C-means clustering separating treatment effects and residual disease. Patient $1(\mathrm{a}, \mathrm{b})$ and patient $3(\mathrm{c}, \mathrm{d})$ are shown. Figures a,c show the results obtained when only including post-LITT MRI feature, whereas Figures b, d show the improvement obtained by incorporating the 10 highest scoring features (Table 2). Residual disease in blue, ablated area in purple. The residual disease area is also indicated with a yellow arrow.

increased substantially, while it has decreased in the residual disease. The reason time-to-peak reduces in residual disease might be reduced is due to inflammation effects or the cancer needing more nutrients to recover from the effects of the treatment. As scar tissue also has very limited diffusivity (similar to prostate cancer lesion), the diffusion-weighted imaging might be less useful to assess treatment response, even though it is a very important modality for initial diagnosis of prostate cancer. Compared to the results of Viswanath et al., ${ }^{6}$ we find that diffusion-weighted features seem of less importance. They compared the ablation zone to normal prostate tissue and found diffusion-weighted feature to be most discriminative. In our case, comparing residual disease to successfully ablated tissue, diffusion-weighted features do not seem important. This indicates that, relative to normal tissue, there is a change in diffusion-weighted features, however, that this change is similar in residual disease and successfully ablated tissue. This again shows that diffusion-weighted imaging might be less useful for detection of residual disease.

\subsection{Objective 3: combine computer-extracted features with feature change to better discriminate residual disease from successfully ablated tissue}

For objective (3) we show that combining the computer-extracted features with the relative feature differences, we improved detection of residual disease, which is presented quantitatively in Table 3, column 2 and qualitatively in figure 6. For all patients we can see that incorporating change in feature values between pre- and post-LITT MRI improves performance substantially. For one patient we were even able to achieve an AUC of 0.97, compared to 0.80 when using only post-LITT MRI features.

These results shows that, using accurate registration of histopathology and the pre- and post-LITT MRI it is possible to identify features which might be useful in tracking treatment success. Furthermore, in the future we might be able to give quantitative guidelines for the treating physician to help him detect the presence of residual disease and predict patient outcome after successful ablation. 


\section{CONCLUDING REMARKS AND CONTRIBUTIONS}

A unique clinical trial at the Radboud University Medical Center, in which 4 patients underwent a prostatectomy after LITT treatment, yielded ex-vivo histopathologic specimens along with pre- and post-LITT MRI. In this work we used this data to address a unique set of questions: (1) are there imaging characteristics specific for residual disease and treatment effects? (2) Can we identify features which showed the most treatment related change relative to residual disease? And (3) can we improve detection of residual disease by incorporating feature change in addition to post-LITT MRI features? By co-registering histology, pre- and post-LITT MRI we were

\begin{tabular}{l|c|c|}
\hline Patient & Features & Features + feature differences \\
\hline Patient 1 & 0.80 & 0.97 \\
Patient 2 & 0.62 & 0.69 \\
Patient 3 & 0.69 & 0.78 \\
\hline
\end{tabular}

Table 3. Area under the curve on a voxel-by-voxel basis for detecting residual disease. The second column shows the effect of adding the differences between pre- and post-LITT MRI for the 10 scoring features as extra features (Table 2).

able to identify features which (1) differentially express between residual disease and treatment effects (AUC up to 0.80 , Table 3 ). However, the results over all three patients showed that more information is needed to increase accuracy in the detection of residual disease, for example by incorporating feature change.

The main take-aways of this study are:

- The differences between residual disease and successfully ablated tissue cannot be fully characterized by taking into account just the post-LITT MRI

- Different changes in features can be observed between pre- and post-LITT MRI for residual disease and successfully ablated tissue

- Incorporating feature changes as extra features discriminating residual disease and successfully ablated tissue results in a markedly improved detection of residual disease

- Diffusion-weighted imaging seems less important in assessing therapy effects compared to dynamic contrastenhanced or T2-weighted imaging.

Our study also has several limitations. The most important one is the lack of additional patient data. Currently, the initial results of the clinical trial are being investigated and will hopefully lead to an extended clinical trial. This will allow us to perform statistical tests to assess the significance of our results. Additionally, this will open up the opportunity to do a supervised classification of residual disease on the post-LITT MRI, which is now limited due to the substantial differences in treatment response in the three patients. Finally, the pathology MRI/co-registration is on a $2 \mathrm{D}$ basis whereas a fully $3 \mathrm{D}$ pipeline would most likely further improve results. In future work we intend to evaluate our data on a larger cohort and investigate the use of supervised classification over clustering.

\section{Acknowledgments}

Research reported in this publication was supported by the National Cancer Institute of the National Institutes of Health under award numbers R01CA136535-01, R01CA140772-01, and R21CA167811-01; the National Institute of Diabetes and Digestive and Kidney Diseases under award number R01DK098503-02, the DOD Prostate Cancer Synergistic Idea Development Award (PC120857); the QED award from the University City Science Center and Rutgers University, the Ohio Third Frontier Technology development Grant. The content is solely the responsibility of the authors and does not necessarily represent the official views of the National Institutes of Health. 


\section{REFERENCES}

[1] Hegarty, J., Beirne, P. V., Walsh, E., Comber, H., Fitzgerald, T., and Wallace Kazer, M., "Radical prostatectomy versus watchful waiting for prostate cancer," Cochrane Database Syst Rev, CD006590 (2010).

[2] Rosenkrantz, A. B., Scionti, S. M., Mendrinos, S., and Taneja, S. S., "Role of MRI in minimally invasive focal ablative therapy for prostate cancer," AJR Am J Roentgenol 197, W90-W96 (2011).

[3] Oto, A., Sethi, I., Karczmar, G., McNichols, R., Ivancevic, M. K., Stadler, W. M., Watson, S., and Eggener, S., "MR imaging-guided focal laser ablation for prostate cancer: phase I trial," Radiology 267, 932-940 (2013).

[4] Ahmed, H. U., Arya, M., Freeman, A., and Emberton, M., "Do low-grade and low-volume prostate cancers bear the hallmarks of malignancy?," Lancet Oncol 13, e509-e517 (Nov 2012).

[5] Roberts, H. R., Paley, M., Hall-Craggs, M. A., Lees, W. R., Friedman, E. P., Clemence, M., Buonacorssi, G., and Bown, S. G., "Dynamic magnetic resonance control of interstitial laser photocoagulation therapy of colorectal hepatic metastases," Lancet 343, 1221 (1994).

[6] Viswanath, S., Toth, R., Rusu, M., Sperling, D., Lepor, H., Futterer, J., and Madabhushi, A., "Quantitative evaluation of treatment related changes on multi-parametric MRI after laser interstitial thermal therapy of prostate cancer," in [Proceedings of the SPIE], 86711F-86711F, International Society for Optics and Photonics (2013).

[7] Pickett, B., Kurhanewicz, J., Coakley, F., Shinohara, K., Fein, B., and Roach, 3rd, M., "Use of MRI and spectroscopy in evaluation of external beam radiotherapy for prostate cancer," Int J Radiat Oncol Biol Phys 60, 1047-1055 (2004).

[8] Song, I., Kim, C. K., Park, B. K., and Park, W., "Assessment of response to radiotherapy for prostate cancer: value of diffusion-weighted MRI at 3 t," AJR Am J Roentgenol 194, W477-W482 (2010).

[9] Lindner, U., Lawrentschuk, N., Weersink, R. A., Davidson, S. R. H., Raz, O., Hlasny, E., Langer, D. L., Gertner, M. R., Van der Kwast, T., Haider, M. A., and Trachtenberg, J., "Focal laser ablation for prostate cancer followed by radical prostatectomy: validation of focal therapy and imaging accuracy," Eur Urol 57, 1111-1114 (2010).

[10] Chappelow, J., Bloch, B. N., Rofsky, N., Genega, E., Lenkinski, R., DeWolf, W., and Madabhushi, A., "Elastic registration of multimodal prostate MRI and histology via multiattribute combined mutual information," Med Phys 38, 2005-2018 (2011).

[11] Kitajima, K., Kaji, Y., Fukabori, Y., Yoshida, K., Suganuma, N., and Sugimura, K., "Prostate cancer detection with 3 T MRI: Comparison of diffusion-weighted imaging and dynamic contrast-enhanced MRI in combination with T2-weighted imaging," J Magn Reson Imaging 31, 625-631 (2010).

[12] Barentsz, J. O., Richenberg, J., Clements, R., Choyke, P., Verma, S., Villeirs, G., Rouviere, O., Logager, V., Fütterer, J. J., and European Society of Urogenital Radiology, "ESUR prostate MR guidelines 2012," Eur Radiol 22, 746-757 (2012).

[13] Hambrock, T., Somford, D. M., Huisman, H. J., van Oort, I. M., Witjes, J. A., Hulsbergen-van de Kaa, C. A., Scheenen, T., and Barentsz, J. O., "Relationship between apparent diffusion coefficients at 3.0-T MR imaging and Gleason grade in peripheral zone prostate cancer," Radiology 259, 453-461 (2011).

[14] Vos, E. K., Litjens, G., Kobus, T., Hambrock, T., Kaa, C. A., Barentsz, J. O., Huisman, H., and Scheenen, T. W., "Assessment of prostate cancer aggressiveness using dynamic contrast-enhanced magnetic resonance imaging at 3 t," Eur Urol 64, 448-455 (2013).

[15] Viswanath, S. E., Bloch, N. B., Chappelow, J. C., Toth, R., Rofsky, N. M., Genega, E. M., Lenkinski, R. E., and Madabhushi, A., "Central gland and peripheral zone prostate tumors have significantly different quantitative imaging signatures on 3 tesla endorectal, in vivo T2-weighted MR imagery," $J$ Magn Reson Imaging 36, 213-224 (2012).

[16] Tiwari, P., Kurhanewicz, J., and Madabhushi, A., "Multi-kernel graph embedding for detection, gleason grading of prostate cancer via MRI/mrs," Med Image Anal 17, 219-235 (2013).

[17] Klein, S., Staring, M., Murphy, K., Viergever, M. A., and Pluim, J. P. W., "elastix: a toolbox for intensitybased medical image registration," IEEE Trans Med Imaging 29, 196-205 (2010). 
[18] Litjens, G. J. S., Debats, O. A., van de Ven, W. J. M., Karssemeijer, N., and Huisman, H. J., "A pattern recognition approach to zonal segmentation of the prostate on MRI," in [Med Image Comput Comput Assist Interv], Lect Notes Comput Sci 7511, 413-420 (2012).

[19] Li, Q., Sone, S., and Doi, K., "Selective enhancement filters for nodules, vessels, and airway walls in twoand three-dimensional CT scans," Med Phys 30, 2040-2051 (2003).

[20] Vos, P. C., Barentsz, J. O., Karssemeijer, N., and Huisman, H. J., "Automatic computer-aided detection of prostate cancer based on multiparametric magnetic resonance image analysis," Phys Med Biol 57, 1527-1542 (2012).

[21] Litjens, G., Barentsz, J., Karssemeijer, N., and Huisman, H., "Automated computer-aided detection of prostate cancer in MR images: from a whole-organ to a zone-based approach," in [Medical Imaging], Proceedings of the SPIE 8315, 83150G-83150G-6 (2012).

[22] Li, B., Christensen, G. E., Hoffman, E. A., McLennan, G., and Reinhardt, J. M., "Establishing a normative atlas of the human lung: Intersubject warping and registration of volumetric CT images," Acad Radiol 10, 255-265 (2003).

[23] Tofts, P. S., Brix, G., Buckley, D. L., Evelhoch, J. L., Henderson, E., Knopp, M. V., Larsson, H. B., Lee, T. Y., Mayr, N. A., Parker, G. J., Port, R. E., Taylor, J., and Weisskoff, R. M., "Estimating kinetic parameters from dynamic contrast-enhanced $\mathrm{t}(1)$-weighted MRI of a diffusable tracer: standardized quantities and symbols," J Magn Reson Imaging 10, 223-232 (1999).

[24] Huisman, H. J., Engelbrecht, M. R., and Barentsz, J. O., "Accurate estimation of pharmacokinetic contrastenhanced dynamic MRI parameters of the prostate," J Magn Reson Imaging 13, 607-614 (2001). 